\title{
Is there a value for probiotic supplements in gestational diabetes mellitus? A randomized clinical trial
}

\author{
Neda Dolatkhah', Majid Hajifaraji ${ }^{2 *}$, Fatemeh Abbasalizadeh ${ }^{3}$, Naser Aghamohammadzadeh ${ }^{4}$, \\ Yadollah Mehrabi ${ }^{5}$ and Mehran Mesgari Abbasi ${ }^{6}$
}

\begin{abstract}
Background: Although several studies have found probiotics encouraging in prevention of gestational diabetes mellitus (GDM), the evidence for the use of probiotics in diagnosed GDM is largely limited. The aim of this study was to assess the effect of a probiotic supplement capsule containing four bacterial strains on glucose metabolism indices and weight changes in women with newly diagnosed GDM.

Methods: Sixty-four pregnant women with GDM were enrolled into a double-blind placebo-controlled randomized clinical trial. They were randomly assigned to receive either a probiotic or placebo capsule along with dietary advice for eight consecutive weeks. The trend of weight gain along with glucose metabolism indices was assayed.

Results: During the first 6 weeks of the study, the weight gain trend was similar between the groups. However, in the last 2 weeks of the study, the weight gain in the probiotic group was significantly lower than in the placebo group ( $p<0.05$ ). Fasting blood sugar (FBS) decreased in both intervention (from 103.7 to $88.4 \mathrm{mg} / \mathrm{dl}$ ) and control (from 100.9 to $93.6 \mathrm{mg} / \mathrm{dl}$ ) groups significantly, and the decrease in the probiotic group was significantly higher than in the placebo group $(p<0.05)$. Insulin resistance index in the probiotic group had $6.74 \%$ reduction over the study period $(p<0.05)$. In the placebo group, however, there was an increase in insulin resistance index $(6.45 \%)$, but the observed change in insulin resistance was not statistically significant. Insulin sensitivity index was increased in both groups. The post-intervention insulin sensitivity index in the probiotic group was not significantly different from placebo when adjusted for the baseline levels.
\end{abstract}

Conclusions: The probiotic supplement appeared to affect glucose metabolism and weight gain among pregnant women with GDM. This needs to be confirmed in other settings before a therapeutic value could be approved.

Keywords: Probiotics, Gestational diabetes mellitus, Nutrition, Randomized clinical trial

\section{Background}

Gestational diabetes mellitus (GDM) is a condition in which the pregnant woman has high serum glucose levels during the gestation. It is defined as carbohydrate intolerance first diagnosed during pregnancy [1]. It is characterized by maternal insulin resistance and is associated with inflammation through the gestation [2]. GDM is shown to be associated with a range of adverse

\footnotetext{
* Correspondence: m.hajifaraji@nnftri.ac.ir

${ }^{2}$ Nutrition Society, National Nutrition and Food Technology Research

Institute, Faculty of Nutrition and Food Technology, Beheshti University of Medical Sciences, Baran 3, West Arghavan, Farahzadi Blvd., Shahrak Qods, 19395-4741, istanbul 1981619573, turkey

Full list of author information is available at the end of the article
}

pregnancy outcomes such as preeclampsia and abnormal delivery. The newborns may also suffer from some health problems as a consequence. GDM rates are increasing both in high-income as well as the low- and middle-income countries as a consequence of increasing rates of overweight and obesity [3]. Both women with GDM and their infants are at increased risk of diabetes mellitus and metabolic dysfunction later in life $[4,5]$. It has been confirmed that treatment of GDM improves pregnancy outcomes with significant reductions in the rate of serious perinatal problems such as macrosomia, dystocia and inevitable caesarean section deliveries. 
Generally, the treatment includes diet with or without medication [6].

Probiotics are known to be the good bacteria usually consumed as capsules or drinks to supplement the bowl bacteria. As narrated by Salmin, currently, the most commonly used definition for probiotics is given by Fuller as "Probiotics are live microbial feed supplements which beneficially affect the host animal by improving its intestinal microbial balance" [7, 8]. They are shown to affect the consumer's metabolism. The use of probiotics has been investigated on various infectious or noninfectious conditions [9-13]. Probiotics have also been investigated for their effect on type 2 diabetes or prevention of gestational diabetes [14-21]. Although several studies have found probiotics encouraging in prevention of GDM, the evidence for use of probiotics for those with diagnosed GDM is largely limited. The aim of this study was to assess the effect of a probiotic supplement capsule containing four bacterial strains in comparison with placebo on glucose metabolism indices and weight changes in women with newly diagnosed GDM.

\section{Methods}

In a double-blind placebo-controlled randomized clinical trial, 64 subjects with GDM referred to Alzahra University Hospital in Tabriz, Northwest of Iran, were enrolled during the spring and summer months in 2014. The patients were randomly allocated to receive either probiotic supplement or placebo capsules once daily for 8 weeks.

Each probiotic capsule of four bacterial strains (4 biocap $>4 \times 10^{9} \mathrm{CFU}$ ) in standard freeze-dried culture included Lactobacillus acidophilus LA-5, Bifidobacterium BB-12, Streptococcus thermophilus STY-31 and Lactobacillus delbrueckii bulgaricus LBY-27 plus dextrose anhydrous filler and magnesium stearate lubricant produced by CHR HANSEN, Denmark, packed and gelatin covered in Tehran Darou drug industries.

The eligible subjects to be enrolled included all nulliparous women with GDM screened during 24-28 weeks of gestation who were referred to the specialty and subspecialty gynaecology or endocrinology clinics of Tabriz University of Medical Sciences.

The inclusion criteria were as follows: Nulliparity; Gestational diabetes between 24 and 28 week ( +6 days) of gestation newly diagnosed through screening done by either a gynaecologist or an internal medicine specialist; Age range of 18-45 years; Fasting blood sugar range of 92 to $126 \mathrm{mg} / \mathrm{dl}$ early at the diagnosis; Body mass index (BMI) above $18.5 \mathrm{~kg} / \mathrm{m}^{2}$; No history of type 2 diabetes mellitus; No history of chronic diseases; No smoking and alcohol consumption; Not using probiotic food products during the 2 weeks before intervention; Not using antibiotics during the month before intervention;
Lack of acute gastrointestinal problems a month before trial and Not using Glucocorticoids (GCs) and immunosuppressive drugs. The exclusion criteria were the following: Needing to use insulin of other diabetes drugs through the study period; Use of antibiotics through the study period and Use of GCs and immunosuppressive drugs through the study period.

At baseline, the purpose and method of study were described in detail for the patients, and a trained practitioner provided similar diet recommendations for patients in both groups. Written informed consent was obtained from all the patients for being enrolled into this study. Then, during an interview with the participants, a general questionnaire and a dietary recall questionnaire were completed. The general questionnaire was used to collect data on demographic information, weight before pregnancy, physical activity, past medical history, drug history over the past month and use of probiotic food products over the past 2 weeks before the study. Physical activity was only measured at baseline. Subjects were categorized into three groups: those with low physical activity (those with a sedentary life and physical activity limited to chores such as cooking, sewing, working with computer and so on); those with moderate physical activity whose activity is limited to works such as cleaning or taking care of children and other works needing small amounts of movement or bodily movements and those with high physical activity who used to have brisk walking, running, biking and swimming regularly [22]. A 24$\mathrm{h}$ dietary recall questionnaire was completed at sessions of three nonconsecutive days each (two weekdays and one in weekend) once at the baseline, and then after 4 weeks and also at the end of study. To obtain the nutrient intake of participants based on these 3-day food diaries, we used Nutritionist IV software (First Databank, San Bruno, Calif., USA) modified for Iranian foods. Weight, height and blood pressure were measured, and some information about their dietary habits and weight before pregnancy were also taken. Seca 206 wallmounted stadiometer and Seca 813 digital scale were used to measure weight and height. Body mass index (BMI) was then calculated and categorized according to the world health organization guidelines [23].

After taking blood samples and randomly allocating them into one of two study groups, subjects were given a 2-week package of either probiotic or placebo capsules. Fasting blood samples $(10 \mathrm{ml})$ before and after the intervention were collected from forearm vein by a laboratory technician for measurement of fasting blood glucose and fasting insulin at Alzahra Hospital laboratory. Plasma glucose levels were assessed using a glucose oxidase/peroxidase method as an enzymatic colorimetric (GODPAP) methodology [24] by Pars Azmoon test kits (Pars Azmoon Inc, Tehran, Iran). Serum insulin levels were 
measured by ELISA method using Monobind kit [25]. Homeostasis model assessment insulin resistance (HOMA-IR) was used to assess insulin resistance [26]. A HOMA-IR value above 3.8 is defined as insulin resistance [27]. QUICKI index (quantitative insulin sensitivity check) was used in present study to assess insulin sensitivity [28]. Details of data collection methodology and laboratory testing are published in the trial protocol elsewhere [29].

HOMA-IR was used as the main outcome for estimating the sample size. Sample size was estimated using parameters from the study by Asemi et al. assuming a maximum type 1 error of 0.05 and $90 \%$ statistical power, HOMA-IR index standard deviation equal to $31 \%$ and an effect size equal to 0.2 ; a total number of 32 subjects were estimated to be enrolled for each group taking into account $10 \%$ attrition rate [30]. A total of 64 pregnant women with GDM were randomly allocated using block randomization techniques stratified according to the prepregnancy fasting blood sugar (FBS) and BMI groups. To ensure double blinding, a coder anonymously labelled the capsules packages as "A" or "B" and therapist assigned them according to the random sequence generated through a computer program [31]. Patients were visited in obstetrics and gynaecology clinics of Alzahra University Hospital in Tabriz. Weight and blood pressure measurements were also done every 2 weeks. Through the study course, in order to improve the compliance, the participants were contacted by telephone each week asked about any problems they may have and about gastrointestinal symptoms as well as use of any drugs through the period.

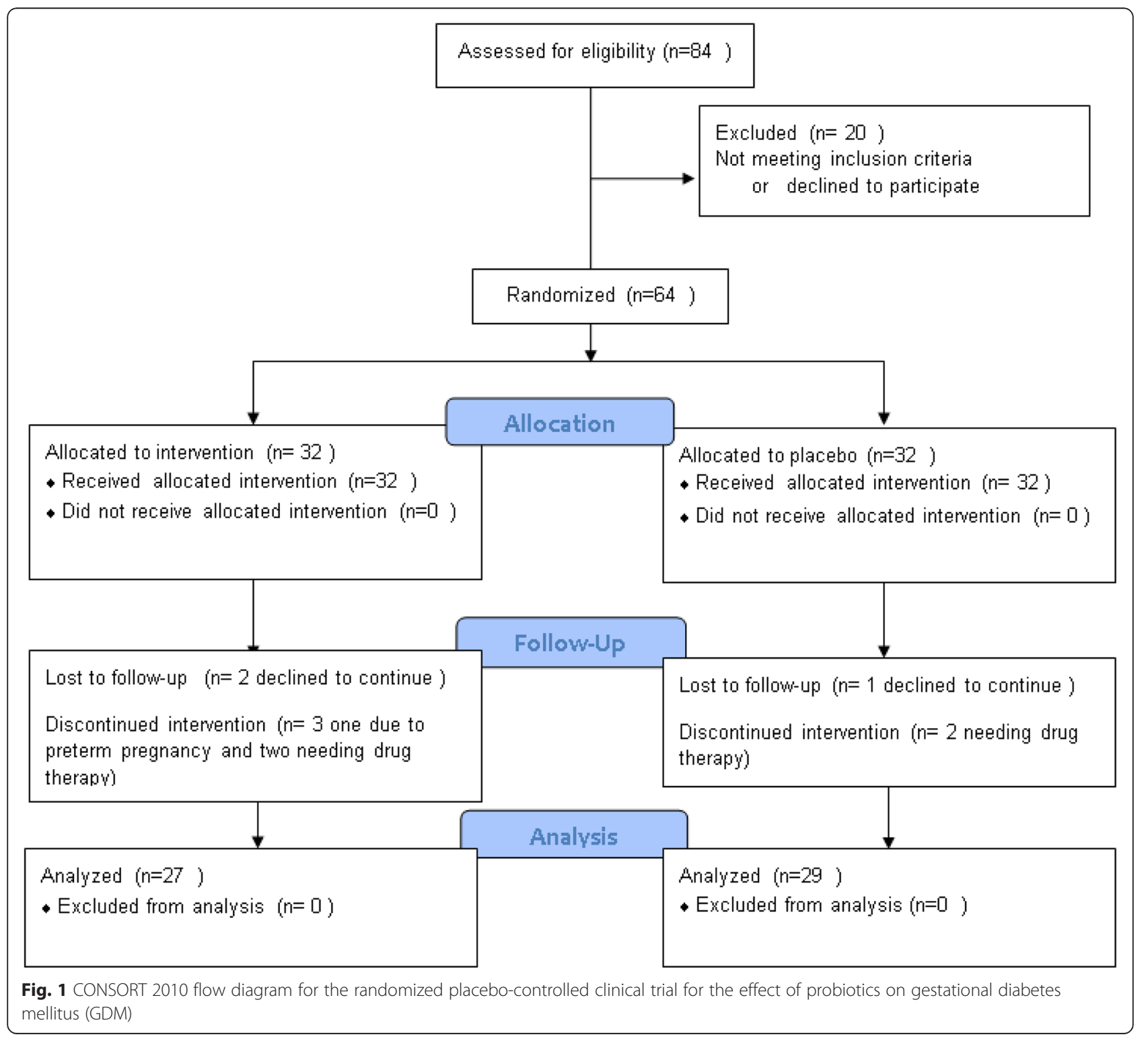


Data were analyzed using SPSS statistical software package version 22. Mean response scales measured over the 8-week study period were analyzed using appropriate statistical methods including independent samples $t$ test, repeated measurements analysis of variance, one-way analysis of variance and analysis of covariance. Energyadjusted nutrient intakes were calculated as the residuals from the regression model, with absolute nutrient intake as the dependent variable and total energy intake as the independent variable. A $p$ value below 0.05 was considered as statistically significant.

\section{Results}

A total of 64 nulliparous pregnant women with gestational diabetes participated in the study, data from the 29 patients in the probiotic group and 27 in the placebo group were finally analyzed (see CONSORT flow diagram in Fig. 1). Mean age of the patients was 27.3 (SD: 5.8) years. Comparison of the baseline characteristics for both groups of pregnant women under study are presented in Table 1.

Comparing the frequency of blood glucose levels at baseline in pregnant women showed that in probiotic group, $69 \%$ of mothers had fasting plasma sugar between 92 and $104 \mathrm{mg} / \mathrm{dl}$ and among $31 \%$ of them, FBS ranged $105-126 \mathrm{mg} / \mathrm{dl}$. The values in the placebo group were 66.7 and $33.3 \%$, respectively. There was no statistically significant difference between the groups in terms of classification of blood glucose at baseline. Mean weight change over the study period is compared between the groups in Fig. 2.

The weight gain over the study period, adjusted also for energy intake, is given in Table 2. It shows that no significant differences between the intervention and control groups were observed in weight gain measures among the pregnant women within 2-week intervals during the first 6 weeks of the study. However, in the last 2 weeks of the study, the weight gain in pregnant women in the probiotic group was significantly lower than in the placebo group. The results stayed statistically significant after adjusting for the changes in daily energy intake between the two groups, $(p<0.05)$.

Fasting blood sugar levels, fasting insulin level, insulin resistance index (HOMA-IR) and insulin sensitivity index (QUICKI index) were not found to be different between the probiotic supplement and placebo groups at baseline. According to the results of the analysis of covariance, $\mathrm{FBS}$ in both the intervention and control groups decreased significantly (14.66 and $7.38 \%$, respectively), both of which were statistically significant ( $p$ $<0.05$ ). Post-intervention comparison, after adjustment for baseline values, showed that decrease in the probiotic group was significantly higher than in the placebo group $(p<0.05)$. Insulin resistance index in the probiotic
Table 1 Baseline comparison of demographic and anthropometric characteristics of the pregnant women with gestational diabetes mellitus enrolled either to receive probiotic or placebo

\begin{tabular}{|c|c|c|c|}
\hline Baseline measures & $\begin{array}{l}\text { Intervention } \\
(N=29)\end{array}$ & $\begin{array}{l}\text { Placebo } \\
(N=27)\end{array}$ & $p$ value \\
\hline Maternal age & $28.14 \pm 6.24$ & $26.48 \pm 5.23$ & 0.36 \\
\hline \multicolumn{4}{|l|}{ Family history of DM } \\
\hline Yes & 16 (55.2\%) & $12(44.4 \%)$ & \multirow[t]{2}{*}{0.59} \\
\hline No & $13(44.8 \%)$ & 15 (55.6 \%) & \\
\hline \multicolumn{4}{|l|}{ Educational level } \\
\hline Under graduate diploma & $4(13.8 \%)$ & $5(18.5 \%)$ & \multirow[t]{3}{*}{0.91} \\
\hline High school diploma & 17 (58.6 \%) & 15 (55.6\%) & \\
\hline Academic education & $8(27.8 \%)$ & $7(25.9 \%)$ & \\
\hline \multicolumn{4}{|l|}{ Employment } \\
\hline Employed & $10(34.5 \%)$ & $9(33.3 \%)$ & \multirow[t]{2}{*}{1.00} \\
\hline Unemployed or housewife & 19 (65.5\%) & 18 (66.7 \%) & \\
\hline \multicolumn{4}{|l|}{ Residence } \\
\hline \multicolumn{4}{|l|}{ Urban } \\
\hline \multirow[t]{2}{*}{ Rural } & 17 (58.6 \%) & 15 (55.6 \%) & \multirow[t]{2}{*}{1.00} \\
\hline & $12(41.4 \%)$ & $12(44.4 \%)$ & \\
\hline \multicolumn{4}{|l|}{ Physical activity } \\
\hline Low & $22(75.9 \%)$ & 17 (63.0 \%) & \\
\hline Moderate & $7(24.1 \%)$ & 10 (37.0 \%) & 0.38 \\
\hline High & 0 & 0 & \\
\hline Weight (kg) & $83.27 \pm 12.06$ & $78.67 \pm 11.09$ & 0.14 \\
\hline Height (cm) & $162.68 \pm 5.65$ & $162.14 \pm 5.93$ & 0.72 \\
\hline Body mass index $\left(\mathrm{kg} / \mathrm{m}^{2}\right)$ & $31.41 \pm 3.92$ & $29.86 \pm 3.39$ & 0.12 \\
\hline
\end{tabular}

Numeric scales are reported as mean \pm standard deviation, and categorical measures are reported as frequency (percent)

group significantly decreased over the study period (6.74 \% reduction), which was statistically significant ( $p$ $<0.05)$. In the placebo group, however, there was an increase in insulin resistance $(6.45 \%)$, but the change was not statistically significant. Insulin sensitivity index was increased in both groups. These changes in the probiotic group $(5.76 \%)$ were statistically significant. The postintervention insulin sensitivity index in the probiotic group was not significantly different from that in the placebo group when adjusted for the baseline levels. Detailed information on changes in glucose metabolism indices are provided in Table 3.

\section{Discussion}

It was observed in present study that the weight gain did not change during the first 6 weeks of the study, but, in the last 2 weeks of the study, the weight gain in pregnant women in the probiotic group was significantly lower than in the placebo group. With respect to glucose metabolism indices, probiotic supplementation was also 


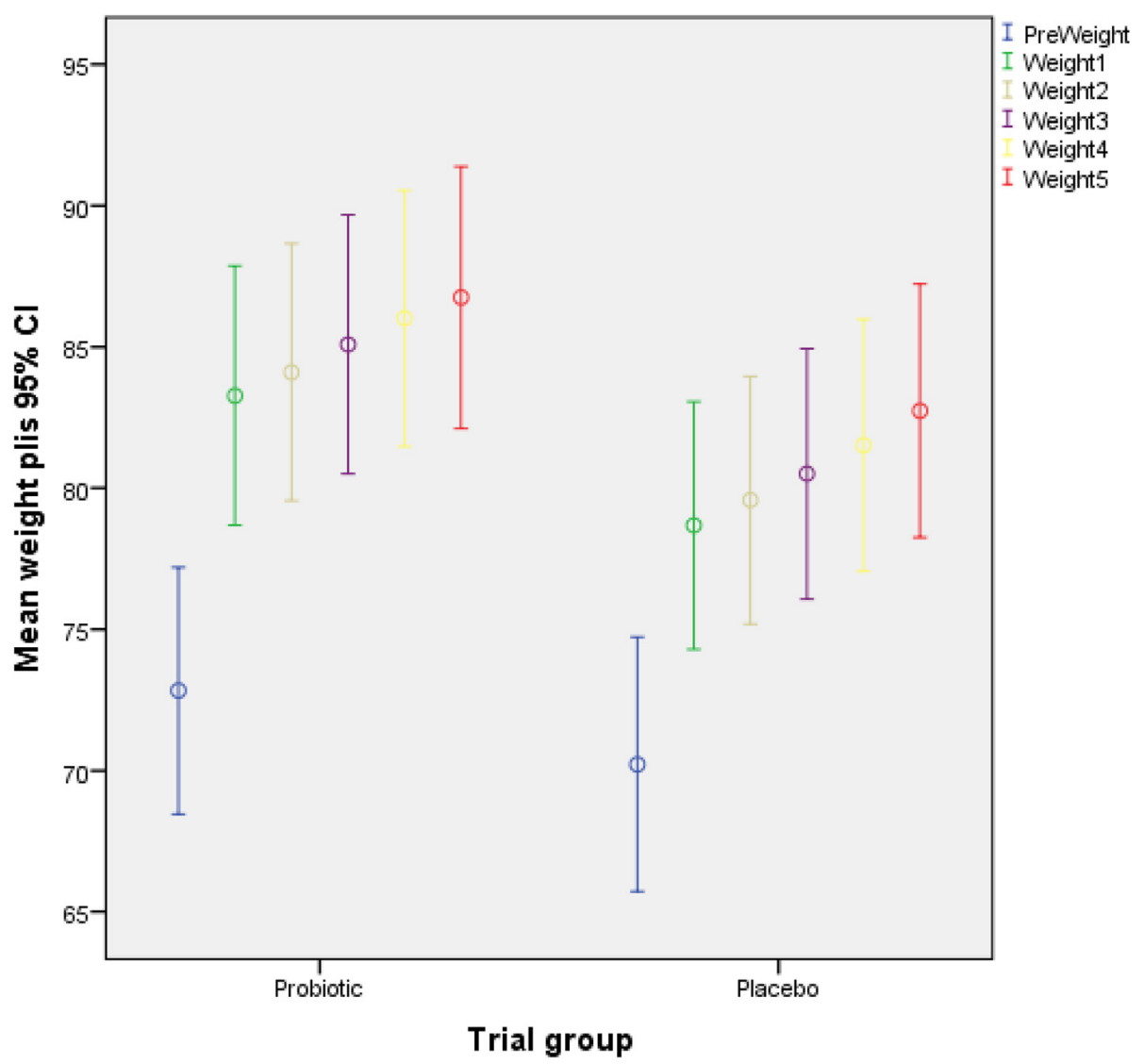

Fig. 2 Mean weight change of women with gestational diabetes mellitus (GDM) over the 8-week study period compared between the probiotic and placebo groups

effective. FBS decreased in the probiotic group significantly more than in the placebo group. Insulin resistance index in the probiotic group significantly decreased over the study period (6.74 \% reduction), which was statistically significant while the increase in insulin resistance $(6.45 \%)$ in the placebo group was not statistically significant. Insulin sensitivity index was increased in both groups, but the post-intervention insulin sensitivity index in the probiotic group was not significantly different from the placebo group.
Although several studies have been done on effect of probiotics on preventing GDM and some studies have been done on general glucose patterns among normal pregnant populations, studies are scarce investigating the effect of probiotics when taken after diagnosis of GDM. One very recent double-blind randomized clinical trial on women with a new diagnosis of GDM and impaired glucose tolerance assigned them to a daily probiotic (Lactobacillus salivarius UCC118) or placebo capsule. Among the 149 women enrolled, no difference

Table 2 Weight gain trend over the 8-week study period comparing women gestational diabetes mellitus receiving probiotic supplement versus placebo capsules

\begin{tabular}{|c|c|c|c|c|c|}
\hline Weight gain & Probiotic $(n=29)$ & Placebo $(n=27)$ & Mean difference $(95 \% \mathrm{Cl})$ & $p$ value ${ }^{*}$ & $p$ value ${ }^{* *}$ \\
\hline First 2 weeks (mean \pm SE) & $0.83 \pm 0.08$ & $0.89 \pm 0.07$ & $-0.61(-0.29,0.17)$ & 0.59 & 0.34 \\
\hline Second 2 weeks (mean \pm SE) & $0.98 \pm 0.07$ & $0.93 \pm 0.06$ & $0.05(-0.14,0.25)$ & 0.60 & 0.69 \\
\hline Third 2 weeks (mean \pm SE) & $0.91 \pm 0.06$ & $1.00 \pm 0.05$ & $-0.09(-0.27,0.08)$ & 0.31 & 0.19 \\
\hline Fourth 2 weeks (mean \pm SE) & $0.74 \pm 0.14$ & $1.22 \pm 0.11$ & $-0.48(-0.85,-0.10)$ & 0.01 & 0.02 \\
\hline First 4 weeks (mean \pm SE) & $1.82 \pm 0.12$ & $1.82 \pm 0.12$ & $-0.008(-0.37,0.35)$ & 0.96 & 0.62 \\
\hline Second 4 weeks (mean \pm SE) & $1.65 \pm 0.13$ & $2.22 \pm 0.13$ & $-0.57(-0.95,-0.19)$ & 0.004 & 0.004 \\
\hline
\end{tabular}

* Independent samples $t$ test

**One-way ANOVA with differences in energy intake as covariate 
Table 3 Changes in glucose metabolism indices among women with gestational diabetes receiving probiotic supplement versus placebo

\begin{tabular}{|c|c|c|c|c|}
\hline Glucose metabolism indices & Probiotic $(n=29)$ & Placebo $(n=27)$ & $p$ value (Independent samples $t$ test) & $p$ value (ANCOVA) \\
\hline \multicolumn{5}{|l|}{ Fasting blood sugar (mg/dl) } \\
\hline Before trial & $103.65(1.34)$ & $100.89(1.52)$ & 0.17 & \multirow[t]{5}{*}{0.02} \\
\hline After trial & $88.37(2.05)$ & $93.59(3.61)$ & 0.20 & \\
\hline$P$ (paired samples $t$ test) & $<0.001$ & 0.02 & & \\
\hline Absolute change & $-15.27(1.83)$ & $-7.30(3.04)$ & 0.02 & \\
\hline Relative change & $-14.66(1.77)$ & $-7.38(15.09)$ & 0.03 & \\
\hline \multicolumn{5}{|l|}{ Fasting serum insulin ( $\mu \mathrm{lU} / \mathrm{ml})$} \\
\hline Before trial & $5.95(0.50)$ & $5.60(0.37)$ & 0.58 & \multirow[t]{5}{*}{0.09} \\
\hline After trial & $5.15(0.41)$ & $6.12(0.50)$ & 0.14 & \\
\hline$P$ (paired samples $t$ test) & 0.16 & 0.30 & & \\
\hline Absolute difference & $-0.80(0.56)$ & $0.52(0.49)$ & 0.08 & \\
\hline Relative difference & $10.55(18.55)$ & $14.55(9.21)$ & 0.85 & \\
\hline \multicolumn{5}{|l|}{ HOMA-IR index } \\
\hline Before trial & $1.52(0.12)$ & $1.38(0.08)$ & 0.41 & \multirow[t]{5}{*}{0.03} \\
\hline After trial & $1.11(0.09)$ & $1.40(0.11)$ & 0.06 & \\
\hline$P$ (paired samples $t$ test) & 0.007 & 0.93 & & \\
\hline Absolute difference & $-0.40(0.13)$ & $0.01(0.12)$ & 0.03 & \\
\hline Relative difference & $-6.74(14.84)$ & $6.45(9.22)$ & 0.46 & \\
\hline \multicolumn{5}{|l|}{ QUICKI index } \\
\hline Before trial & $0.15(0.00)$ & $0.15(0.00)$ & 0.92 & \multirow[t]{5}{*}{0.11} \\
\hline After trial & $0.16(0.00)$ & $0.16(0.00)$ & 0.11 & \\
\hline$P$ (paired samples $t$ test) & 0.02 & 0.46 & & \\
\hline Absolute difference & $0.008(0.003)$ & $0.002(0.002)$ & 0.16 & \\
\hline Relative difference & $5.76(2.16)$ & $1.38(1.69)$ & 0.12 & \\
\hline
\end{tabular}

HOMA-IR homeostasis model assessment insulin resistance, QUICKI quantitative insulin sensitivity check

was observed between the probiotic and placebo groups in post-intervention fasting glucose levels. However, they found a likely effect for the probiotic on lipid profile of the patients [32]. Regardless of the differences in study design and population, the main characteristic possibly describing the reason for positive results in present study may be attributed to different probiotic contents of the supplement in two studies. Observing the effect of probiotics in present study with a smaller sample size while yielding reasonably narrower confidence interval justifies a promising effect for the intervention used in our study. Aside from the different probiotic in present study and the magnitude of potential random error attributable to the sample size, other issues may affect the results either such as the variations in normal weight gain through the pregnancy, variations in gut flora, physical activity and diet. Normally, such variations are controlled through randomization, but this cannot be fully guaranteed unless the study is large enough to ensure the efficacy of randomization [33]. No doubt physical activity is a major risk indicator in several noncommunicable diseases including diabetes. In present study, the physical activity was measured only at baseline, and we recommend to consider it as a time-dependent variable in future clinical trial studies assessing the role of probiotics on GDM. Studies like present study conducted specifically on patients diagnosed with GDM are quite rare; however, there are bunches of studies on role of probiotics on prevention of GDM or its related pregnancy outcomes. Generally, the effect of probiotics in prevention of GDM and poor pregnancy outcomes have been shown to be promising [6, 20, 21, 34-39]. Probiotics have also been investigated when combined with diet recommendations. In a study from Finland, 256 pregnant women were randomized early through the gestation to receive nutrition counselling or not to receive it as controls; the group with dietary intervention was further randomized to receive probiotics (Lactobacillus rhamnosus GG and Bifidobacterium lactis Bb12; diet/probiotics) or placebo (diet/placebo). It was found 
that blood glucose levels as well as HOMA-IR and QUICKI indices were lowest in the diet/probiotics group concluding that improved blood glucose control could be reached by dietary counselling combined with probiotics even in normoglycaemic pregnant women [40]. A recent review on dietary interventions, lifestyle changes and dietary supplements in preventing GDM has concluded that trials in which only intake or expenditure has been the target of interventions may not achieve positive efficacy results, whereas combined interventions and dietary and lifestyle interventions could show better efficacy in the reduction of GDM prevalence. The same review has stated the results of probiotic studies to be promising and has recommended future larger scale studies [41]. Although, the cost-effectiveness of probiotics has not been well documented in literature, probiotic supplements have been shown to have acceptability among patients $[42,43]$.

About $95 \%$ of the intestinal microbiome in healthy people with normal weight consists of the species belonging to three phyla of the bacteria including Bacteroidetes, Firmicutes and Actinobacteria. The plausibility of the observed effect of probiotics is not much hard to be approved considering the available evidence on role of healthy gut microbiome on carbohydrate metabolism and the synergistic relationships of probiotic foods and supplements with the host gut microbiome [21, 44, 45]. To complete the plausibility pattern, the changes in gut microbiome during diabetes mellitus should also be considered. A new theory suggests that gut microbiota have a role in the regulation of the energy homeostasis and causing metabolic diseases and insulin resistance [46]. It has been shown that type 2 diabetes is associated with changes in gut microbiome. For instance, it has been shown that the ratio of Bacteroidetes to Firmicutes species is associated with diabetes type 2 and FBS, especially that the changes in gut microbiome are different from what is observed during obesity and a larger number of opportunistic pathogens along with a lower number of butyrate-producing bacteria are attributed to presence of diabetes [21, 47-51].

\section{Conclusions}

The probiotic supplement appeared to affect glucose metabolism and weight gain among pregnant women with GDM.

\section{Ethical issues}

Study protocol was approved by the Research Ethics committee of International branch of Shahid Beheshti University of Medical Sciences as a thesis proposal for $\mathrm{PhD}$ degree in Nutrition Sciences. Written informed consent was taken from all participants.

The clinical trial was registered in the Iranian Registry of Clinical Trials with reference number
IRCT201405181597N3, available at: http://www.irct.ir, accessible through world health organization database of clinical trial registries.

\section{Statement of human and animal rights}

All procedures followed were in accordance with the ethical standards of the responsible committee on human experimentation (institutional and national) and with the Helsinki Declaration of 1975, as revised in 2008 (5).

\section{Competing interests}

The authors declare that they have no conflict of interest.

\section{Authors' contributions}

ND was the PhD student doing her thesis and contributed $A-Z$ of research conduct and reporting. $\mathrm{MH}$ was the main supervisor and contributed $\mathrm{A}-\mathrm{Z}$ of research conduct and reporting. FA, NA and YM contributed in clinical examinations and data collection as well as interpretation of the results. MA contributed in laboratory assays. All the authors contributed in drafting or reviewing the manuscript. All authors read and approved the final manuscript.

\section{Acknowledgement}

This paper is taken from PhD Thesis of Nutrition Sciences, International Branch of Shahid Beheshti University of Medical Sciences, Tehran, Iran. We are grateful to all the participants who helped us to conduct this study and Tehran Darou Pharmaceuticals, Tehran, Iran, for providing 4-Biocap (a probiotic supplement) for the present study. The authors would like to thank the staff of the Alzahra Obstetrics and Gynecology Clinic (Tabriz, Iran) for their assistance with this project during the study.

\section{Author details}

${ }^{1}$ Department of Nutrition Sciences, Shahid Beheshti University of Medical Sciences (SBUMS), International Branch, tehran, iran. ${ }^{2}$ Nutrition Society, National Nutrition and Food Technology Research Institute, Faculty of Nutrition and Food Technology, Beheshti University of Medical Sciences, Baran 3, West Arghavan, Farahzadi Blvd., Shahrak Qods, 19395-4741, istanbul 1981619573, turkey. ${ }^{3}$ Department of Obstetrics \& Gynecology, Tabriz University of Medical Sciences, tabriz, iran. ${ }^{4}$ Section of Endocrinology \& Metabolism, Department of Internal Medicine, Tabriz University of Medical Sciences, Tabriz, Iran. ${ }^{5}$ Department of Biostatistics \& Epidemiology, Shahid Beheshti University of Medical Sciences, Tehran, Iran. ${ }^{6}$ Drug Applied Research Center, Tabriz University of Medical Sciences, Tabriz, Iran.

Received: 22 May 2015 Accepted: 16 November 2015

Published online: 25 November 2015

\section{References}

1. Hadar E, Oats J, Hod M. Towards new diagnostic criteria for diagnosing GDM: the HAPO study. J Perinat Med. 2009:37:447-9.

2. Kuhl C. Etiology and pathogenesis of gestational diabetes. Diabetes Care. 1998;21 Suppl 2:B19-26.

3. Ma RC, Chan JC. Pregnancy and diabetes scenario around the world: China. Int J Gynaecol Obstet. 2009;104:S42-5.

4. Hossain P, Kawar B, El NM. Obesity and diabetes in the developing world-a growing challenge. N Engl J Med. 2007;356:213-5.

5. Moore TR. Fetal exposure to gestational diabetes contributes to subsequent adult metabolic syndrome. Am J Obstet Gynecol. 2010;202:643-9.

6. Barrett HL, Dekker NM, Conwell LS, Callaway LK. Probiotics for preventing gestational diabetes. Cochrane Database Syst Rev. 2014;2, CD009951.

7. Salminen, Ouwehand A, Benno Y, Lee YK. Probiotics: how should they be defined? Trends Food Sci Technol. 1999;10:107-10.

8. Fuller R. Probiotics in man and animals. J Appl Bacteriol. 1989;66:365-78.

9. Saez-Lara MJ, Gomez-Llorente C, Plaza-Diaz J, Gil A. The role of probiotic lactic acid bacteria and bifidobacteria in the prevention and treatment of inflammatory bowel disease and other related diseases: a systematic review of randomized human clinical trials. Biomed Res Int. 2015;2015:505878.

10. Allen SJ. The potential of probiotics to prevent Clostridium difficile infection. Infect Dis Clin North Am. 2015;29:135-44. 
11. Guandalini S. Are probiotics or prebiotics useful in pediatric irritable bowel syndrome or inflammatory bowel disease? Front Med (Lausanne). 2014;1:23.

12. Tang RB, Chang JK, Chen HL. Can probiotics be used to treat allergic diseases? J Chin Med Assoc. 2015;78:154-7.

13. Ruggiero P. Use of probiotics in the fight against Helicobacter pylori. World J Gastrointest Pathophysiol. 2014;5:384-91.

14. Mazloom Z, Yousefinejad A, Dabbaghmanesh MH. Effect of probiotics on lipid profile, glycemic control, insulin action, oxidative stress, and inflammatory markers in patients with type 2 diabetes: a clinical trial. Iran J Med Sci. 2013;38:38-43.

15. Alokail MS, Sabico S, Al-Saleh Y, Al-Daghri NM, Alkharfy KM, Vanhoutte PM, et al. Effects of probiotics in patients with diabetes mellitus type 2: study protocol for a randomized, double-blind, placebo-controlled trial. Trials. 2013:14:195.

16. Frontiers in Endocrinology Editorial Office. Retraction: colonic flora, probiotics, obesity and diabetes. Front Endocrinol (Lausanne). 2013:4:110.

17. Gomes AC, Bueno AA, de Souza RG, Mota JF. Gut microbiota, probiotics and diabetes. Nutr J. 2014;13:60.

18. Sekhar MS, Unnikrishnan MK. Probiotic research for diabetes prevention. Nutrition. 2015;31:248.

19. Panwar H, Rashmi HM, Batish VK, Grover S. Probiotics as potential biotherapeutics in the management of type 2 diabetes-prospects and perspectives. Diabetes Metab Res Rev. 2013;29:103-12.

20. Nitert MD, Barrett HL, Foxcroft K, Tremellen A, Wilkinson S, Lingwood B, et al. SPRING: an RCT study of probiotics in the prevention of gestational diabetes mellitus in overweight and obese women. BMC Pregnancy Childbirth. 2013;13:50.

21. Barrett HL, Callaway LK, Nitert MD. Probiotics: a potential role in the prevention of gestational diabetes? Acta Diabetol. 2012;49 Suppl 1:S1-S13.

22. Mahan LK, Escott-Stump S, Raymond JL. Krause's food \& the nutrition care process. 13th ed. 2012

23. Diet, nutrition and the prevention of chronic diseases. World Health Organ Tech Rep. Ser. 2003;916:i-viii, 1-149. 2003. Ref Type: Report

24. Barham D, Trinder P. An improved colour reagent for the determination of blood glucose by the oxidase system. Analyst. 1972;97:142-5.

25. MacDonald MJ, Gapinski JP. A rapid ELISA for measuring insulin in a large number of research samples. Metabolism. 1989;38:450-2.

26. Matthews DR, Hosker JP, Rudenski AS, Naylor BA, Treacher DF, Turner RC Homeostasis model assessment: insulin resistance and beta-cell function from fasting plasma glucose and insulin concentrations in man. Diabetologia. 1985;28:412-9.

27. Bodlaj G, Berg J, Pichler R, Biesenbach G. Prevalence, severity and predictors of HOMA-estimated insulin resistance in diabetic and nondiabetic patients with end-stage renal disease. J Nephrol. 2006;19:607-12.

28. Hrebicek J, Janout V, Malincikova J, Horakova D, Cizek L. Detection of insulin resistance by simple quantitative insulin sensitivity check index QUICKI for epidemiological assessment and prevention. J Clin Endocrinol Metab. 2002; 87:144-7.

29. Dolatkhah N, Hajifaraji M, Abbasalizadeh F, Aghamohammadzadeh N, Mehrabi Y, Megari Abbasi M: Effect of probiotics in gestational diabetes mellitus: study protocol. Journal of Clinical Research \& Governance 2015, Unplublished.

30. Asemi Z, Samimi M, Tabassi Z, Naghibi RM, Rahimi FA, Khorammian H, et al. Effect of daily consumption of probiotic yoghurt on insulin resistance in pregnant women: a randomized controlled trial. Eur J Clin Nutr. 2013;67:71-4.

31. Asghari-Jafarabadi M, Sadeghi-Bazargani H. Randomization: techniques and software-aided implementation in medical studies. J Clin Res Gov . 2014;3.

32. Lindsay KL, Brennan L, Kennelly MA, Maguire OC, Smith T, Curran S, et al. Impact of probiotics in women with gestational diabetes mellitus on metabolic health: a randomized controlled trial. Am J Obstet Gynecol. 2015.

33. Hajebrahimi S, Mostafaie A, Sadeghi-Bazargani H. Evidence for the future-designing a clinical trial. Indian J Urol. 2011;27:494-7.

34. Luoto R, Laitinen $\mathrm{K}$, Nermes $\mathrm{M}$, Isolauri E. Impact of maternal probioticsupplemented dietary counselling on pregnancy outcome and prenatal and postnatal growth: a double-blind, placebo-controlled study. Br J Nutr. 2010; 103:1792-9.

35. Luoto R, Laitinen $K$, Nermes M, Isolauri E. Impact of maternal probioticsupplemented dietary counseling during pregnancy on colostrum adiponectin concentration: a prospective, randomized, placebo-controlled study. Early Hum Dev. 2012;88:339-44.
36. Lindsay KL, Walsh CA, Brennan L, McAuliffe FM. Probiotics in pregnancy and maternal outcomes: a systematic review. J Matern Fetal Neonatal Med. 2013;26:772-8.

37. Lindsay KL, Kennelly M, Culliton M, Smith T, Maguire OC, Shanahan F, et al. Probiotics in obese pregnancy do not reduce maternal fasting glucose: a double-blind, placebo-controlled, randomized trial (Probiotics in Pregnancy Study). Am J Clin Nutr. 2014;99:1432-9.

38. Gomez Arango LF, Barrett HL, Callaway LK, Nitert MD. Probiotics and pregnancy. Curr Diab Rep. 2015;15:567.

39. Rogozinska E, Chamillard M, Hitman GA, Khan KS, Thangaratinam S. Nutritional manipulation for the primary prevention of gestational diabetes mellitus: a meta-analysis of randomised studies. PLoS One. 2015;10, e0115526.

40. Laitinen K, Poussa T, Isolauri E. Probiotics and dietary counselling contribute to glucose regulation during and after pregnancy: a randomised controlled trial. Br J Nutr. 2009;101:1679-87.

41. Facchinetti F, Dante G, Petrella E, Neri I. Dietary interventions, lifestyle changes, and dietary supplements in preventing gestational diabetes mellitus: a literature review. Obstet Gynecol Surv. 2014;69:669-80.

42. Lindsay KL, Brennan L, McAuliffe FM. Acceptability of and compliance with a probiotic capsule intervention in pregnancy. Int J Gynaecol Obstet. 2014; 125:279-80.

43. Arslan S, Durak AN, Erbas M, Tanriverdi E, Gulcan U. Determination of microbiological and chemical properties of probiotic boza and its consumer acceptability. J Am Coll Nutr. 2015;34:56-64.

44. Arumugam M, Raes J, Pelletier E, Le PD, Yamada T, Mende DR, et al. Enterotypes of the human gut microbiome. Nature. 2011;473:174-80.

45. Greiner T, Backhed F. Effects of the gut microbiota on obesity and glucose homeostasis. Trends Endocrinol Metab. 2011;22:117-23.

46. Moreno-Indias I, Cardona F, Tinahones FJ, Queipo-Ortuno MI. Impact of the gut microbiota on the development of obesity and type 2 diabetes mellitus. Front Microbiol. 2014;5:190.

47. Sun Q, Wedick NM, Pan A, Townsend MK, Cassidy A, Franke AA, et al. Gut microbiota metabolites of dietary lignans and risk of type 2 diabetes: a prospective investigation in two cohorts of U.S. women. Diabetes Care. 2014;37:1287-95.

48. Larsen N, Vogensen FK, van den Berg FW, Nielsen DS, Andreasen AS, Pedersen BK, et al. Gut microbiota in human adults with type 2 diabetes differs from non-diabetic adults. PLoS One. 2010;5, e9085.

49. Burcelin R, Serino M, Chabo C, Blasco-Baque V, Amar J. Gut microbiota and diabetes: from pathogenesis to therapeutic perspective. Acta Diabetol. 2011; 48:257-73.

50. Qin J, Li Y, Cai Z, Li S, Zhu J, Zhang F, et al. A metagenome-wide association study of gut microbiota in type 2 diabetes. Nature. 2012;490:55-60.

51. Esteve E, Ricart W, Fernandez-Real JM. Gut microbiota interactions with obesity, insulin resistance and type 2 diabetes: did gut microbiote co-evolve with insulin resistance? Curr Opin Clin Nutr Metab Care. 2011;14:483-90.

\section{Submit your next manuscript to BioMed Central and we will help you at every step:}

- We accept pre-submission inquiries

- Our selector tool helps you to find the most relevant journal

- We provide round the clock customer support

- Convenient online submission

- Thorough peer review

- Inclusion in PubMed and all major indexing services

- Maximum visibility for your research

Submit your manuscript at www.biomedcentral.com/submit 